examining the impact of these clinic-level factors and individuallevel uptake of these services on RIC are needed.

3462

\section{Community-Engaged Research in Emergency Dispatch: Getting a 360 View}

Alissa L Wheeler ${ }^{1}$, Heather Darata and Jenny Hurst

${ }^{1}$ International Academies of Emergency Dispatch

OBJECTIVES/SPECIFIC AIMS: Community-engaged research can provide important input to researchers to understand the impact of health services on diverse communities. In emergency (911) dispatch research, most studies have focused on specific health conditions, especially on identifying and managing those conditions remotely and identifying the most appropriate resources to send. Community-engaged research can add a needed component to these studies, identifying not only what happens when someone calls 911, but who calls and who doesn't, what barriers community members encounter when they call, and what they expect from their 911 service. METHODS/STUDY POPULATION: A recent study published in the Journal of Clinical and Translational Science outlined a method for identifying and evaluating the needed competencies and readiness of individual researchers to do effective communityengaged research. The investigators involved in an ongoing study on community attitudes toward 911 propose to use the methods outlined in that study to receive feedback from their Community Advisory Board on their own competencies and readiness. RESULTS/ANTICIPATED RESULTS: It is anticipated that 13 people will be involved in providing feedback to the investigators, including all official member of the Community Advisory Boards and all supportive academic staff and faculty. The feedback will be gathered using a survey instrument developed from the recentlypublished study and will include questions about the purpose of the research, openness to feedback, communication, cultural sensitivity, community presence, power sharing, recognizing partner contributions, and developing community capacity. DISCUSSION/ SIGNIFICANCE OF IMPACT: Identifying the most appropriate resources to send to any given emergency is the primary role of the emergency dispatcher. However, they are also public servants, providing care and comfort in a time of stress to members of many diverse communities. As such, it is critical that they understand the needs and expectations of those communities, as well as the barriers they face in calling 911. The proposed study adds value to an ongoing community-engaged research project by providing feedback about readiness and competency to the investigators.

\section{Critical Barriers to Effective Community-Academic} Research Partnerships and Potential Solutions

Susan J Woolford, Ayse G. Buyuktur, Patricia Piechowski, Aalap Doshi and Erica E. Marsh

University of Michigan School of Medicine

OBJECTIVES/SPECIFIC AIMS: Background: The importance of engaging community in research and fostering community-academic research partnerships is increasingly acknowledged by Clinical and Translational Science Award (CTSA) institutes. However, forming and maintaining such collaborations is often hampered by numerous challenges. It is critical to investigate the barriers to effective community-academic partnerships and to develop novel approaches to overcome these barriers. Objective: To explore community and academic perspectives of the challenges faced by community-academic research partnerships and potential solutions to these identified challenges. METHODS/STUDY POPULATION: Methods: In an effort to explore creative approaches to address these issues, the Community Engagement Program at the Michigan Institute for Clinical \& Health Research (MICHR), the CTSA site that serves Michigan, hosted a retreat to elicit the input of community members and academics from across the state. There was a mix of participants ranging from those with established community-academic partnerships to others who were new to community-engaged research and in early stages of forming partnerships. At the retreat, attendees were randomly divided into groups and asked to answer the specific question, "What are your barriers to partnering in research?" After each group identified a set of barriers and reported their findings to the entire room, attendees were asked to work again in their small groups to discuss potential solutions to these barriers. Ideas for solutions were also shared with the entire room. As part of the process of brainstorming about these questions, attendees were asked to document their ideas - for both barriers and solutions - on post-it notes which were then grouped by category. Artifacts from the retreat were saved digitally and transcripts made from these records. The findings were then analyzed to identify common themes. RESULTS/ANTICIPATED RESULTS: Results: Eighty-six participants attended the retreat from across the state of Michigan. Fortythree represented community organizations that focus on addressing a wide array of social determinants of health issues. The remaining forty-three participants represented various academic institutions. The most frequently mentioned challenges to community-academic partnerships were related to communication and relationship building. To overcome barriers in these areas, participants noted that it is critical to collaboratively and explicitly identify shared goals, values and norms in the early stages of partnership development. This was closely linked to the need for additional funding to help foster and strengthen relationships by allowing partners to spend time together to both work and socialize informally, preferably in face-to-face settings. These were deemed crucial for building trust and common ground. In addition, more equitable funding and role distribution including shared leadership and governance of research projects between community and academia - that recognizes and supports the true costs of involvement in research for community members was viewed as important. Other frequently noted issues on the part of community members were the need for greater respect for community partners and for more training opportunities to build capacity within communities to participate in research. Participants from academic institutions emphasized that the current requirements and timeline for promotion in academia make it harder for them to participate in community-engaged research, especially as early career researchers. They maintained that wider recognition of the value of community-engaged research is necessary and that this requires the support of home departments. Finally, participants underscored the importance of building infrastructure to better connect potential partners from the community and academia by making it easier to identify common interests and reciprocal strengths. DISCUSSION/ SIGNIFICANCE OF IMPACT: Conclusion: The problems faced by community-academic partnerships may be alleviated by working with community and academic members to identify potential solutions. Further work is needed to systematically examine barriers and the efficacy of solutions to enhance community-academic partnerships. Acknowledgements: We thank all attendees of the MICHR Community Engagement retreat for their participation in this activity that explored barriers to effective community-academic 
partnerships. Their honest and frank feedback was essential to broaching sensitive topics related to partnership development, and to identify realistic and practical solutions. We also thank all members of the planning committee and our colleagues in the Community Engagement Program for their work on bringing together community and academic members for this retreat. This project was supported by grant number UL1TR002240 from the National Center for Advancing Translational Sciences (NCATS).

\section{Developing a REDCap Database to Understand Partnership Cultivation Efforts}

Grisel M. Robles-Schrader ${ }^{1}$, Gina Curry, Josefina Serrato, Jen Brown and Keith A Herzog

${ }^{1}$ Northwestern University

OBJECTIVES/SPECIFIC AIMS:.Outline the development and purpose of the partnership brokering database in REDCap. Provide an overview of the tool and how it works. Discuss how this tool facilitates partnership-brokering activities and discuss plans for future use METHODS/STUDY POPULATION: Research Electronic Data Capture (REDCap) is a secure, web-based application developed at Vanderbilt University to assist with systematic data management of small and medium sized projects. CCH utilized REDCap to build a custom data management warehouse entitled the Partnership Brokering Tool. Information compiled in various formats (handwritten notes, spreadsheets, etc.) over the past 10 years by CCH staff, was then systematically organized and entered into the Partnership Brokering Tool. The tool captures information such as individual contact information, organizational affiliation (academic, community, faith, government etc.), research interests (35 categories asthma, diabetes, heart disease, etc.), communities of foci (children, elderly, LGBTQ, ethnicity, etc.), and target geographic community served (Chicago north, south, suburban, Illinois, etc.). RESULTS/ ANTICIPATED RESULTS: Data was compiled on 451 community groups and organizations and 77 partners in academia thus far. Community organizations represent a range of community sectors including advocacy and policy groups, community-based, faithbased organizations, foundations, media, schools, etc. throughout the Chicagoland area. Data analysis activities are underway, however, results will also be shared regarding characteristics of the communities these organizations serve including:. Age range. Special populations (as defined by the CSTI grant). Underrepresented racial and ethnic communities. DISCUSSION/SIGNIFICANCE OF IMPACT: The Partnership Brokering Tool has provided a format for $\mathrm{CCH}$ to systematically gather information about the relationships staff have cultivated with community groups and organizations. Unlike an email management system, this REDCap project is highly useful in capturing the parameters of our partner pool, identifying partnership gaps, and matching individuals interested in collaborating with researchers or community organizations that have a particular skill set or research interest. The Partnership Brokering Tool has also facilitated stakeholder engagement dedicated to guiding the centers' overall goals, objectives, and programming. Finally, utilizing REDCap has streamlined efforts in reporting quantitative and qualitative data about these organizations. In the next phase of this project, $\mathrm{CCH}$ will utilize the database to assess the nature of the relationship between $\mathrm{CCH}$ and community groups and organizations.
Developing Relevant Community Engagement Metrics to Evaluate Engagement Support and Outcomes

Grisel M. Robles-Schrader ${ }^{1}$, Keith A Herzog and Josefina Serrato

${ }^{1}$ Northwestern University

OBJECTIVES/SPECIFIC AIMS: The goals in this project were two-fold: Develop metrics that assessed community engagement support the center provides, and. Systematically document the fluid and time-intensive nature of providing community engaged research support, as well as key outcomes. METHODS/STUDY POPULATION: The CCH utilized REDCap software in combination with Excel, to create and implement a data collection system to monitor and report on the full spectrum of engagement activities offered by the center. Center staff collaborated in identifying relevant metrics, developing the data collection instruments, and beta-testing instruments with real examples. This facilitated the integration of contextual factors (defined as factors such as the history, size, and diversity of the community, the organizational mission, the structure and size of the CE team, the number of years a university has been supporting community-engaged research work, etc.). Taking a collaborative approach in developing the center's evaluation plan offered the added benefit of facilitating staff/faculty buy-in, building staff capacity, and engaging the team in understanding concepts related to performance measurement versus management. RESULTS/ ANTICIPATED RESULTS: Key benefits of these engagement tracking systems include: consolidating data into a central location, standardizing tracking processes and critical definitions, and supporting more automated reporting systems (e.g., dashboards) that facilitate quality improvement and highlight success stories. Data were compiled and reported via on-line dashboard (REDCap and Tableau) to help center leadership and staff analyze:. Quality improvement issues (How quickly are we responding to a request for support? Are we providing resources that meet the needs of community partners? Academics? Community-academic partnerships?);. Qualitative process analysis (In what research phase are we typically receiving requests for support (e.g. proposal development phase, implementation phase, etc.)? What types of projects are applying for seed grants? After the seed grant ends, are the community-academic partnerships continuing to partner on research activities?);. Outcomes (Are new partnerships stemming from our support? Are supported research projects leading to new policies, practices, programs?). DISCUSSION/SIGNIFICANCE OF IMPACT: There is a gap in the literature regarding meaningful, actionable, and feasible community engaged metrics that capture critical processes and outcomes. This project identified many more relevant metrics and demonstrates that it is worthwhile to take a collaborative, inclusive approach to identifying, tracking, and reporting on key process and outcome metrics in order to convey a more comprehensive picture of community engagement activities and to inform continuous improvement efforts. Community engagement centers across CTSIs offer a similar range of programs and services. At the same time, much of the community-engaged research literature describes metrics related to community-academic grant submissions, funds awarded, and peer-reviewed publications. Experts that work in the arena of providing community engagement support recognize that these metrics are sufficient in understanding the spectrum of engagement opportunities. Community engagement (CE) teams nationally can utilize these metrics in developing their evaluation infrastructure. At the national level, NCATS can utilize the metrics for CE common metrics related to these programs and services. Critical to this 